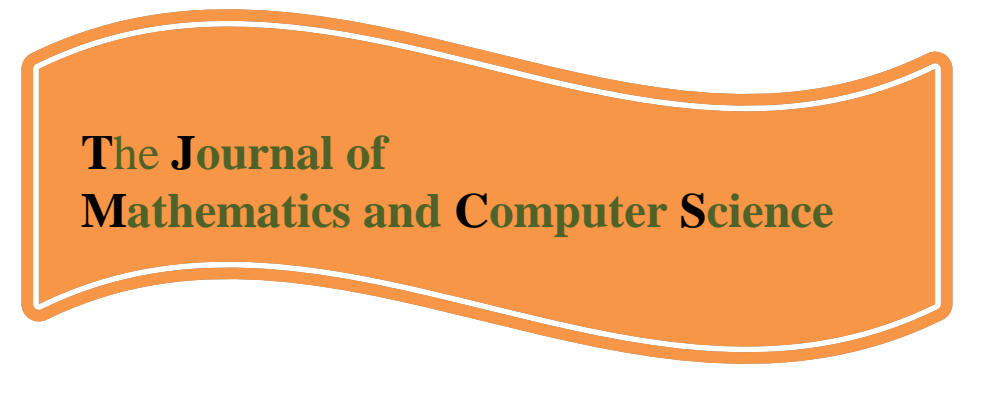

Available online at

\title{
http://www.TIMCS.com
}

The Journal of Mathematics and Computer Science Vol .5 No.4 (2012) 297-303

\section{Designing a Novel Fuzzy Indicator for Predicting Rate of Euro-Dollar in International Foreign Exchange}

\author{
Ahmad J. Afshari \\ Faculty of Engineeing, shomal University, Amol, Iran \\ afshari@shomal.ac.ir \\ Danial Khoonmirzaie \\ Faculty of Engineeing, Payame Nur University of Tehran \\ danielmirzaee@gmail.com

\section{Somaye Rasouli} \\ Faculty of Engineeing, shomal University, Amol, Iran \\ rasouly252@yahoo.com \\ Ensiye Farrokhi \\ Faculty of Engineeing, Payame Nur University of Tehran \\ anis.farrokhi@yahoo.com
}

Received: February 2012, Revised: November 2012

Online Publication: December 2012

\section{Abstract}

Capital market contains different sectors and one of its important sectors is currency market. High turnover in this market has attracted many investors. In this article an efficient system of recognizing point of buy and sell is invented with the help of fuzzy logic. In suggested model functions of triangular and trapezoidal membership is used for defining linguistic variables which can be used for symbol of Euro - Dollar. Inventing system based on fuzzy logic is the first one of its type and its direct application in Forex indicates its efficiency in the real world which can be used in developed trading expert systems for exchange market.

Keywords: Forex; Auto trading; MQL language; Fuzzy indicator.

\section{Mathematics Subject Classification: Primary 54A40; Secondary 46S40.}

\section{Introduction.}

Financial markets are formed of different sectors like titles of commodity market, stock, future contract and etc. In these markets depending on their type, different symbols are used for buying and selling. In exchange market symbols available for currency of various countries is varied which 
are measured with each other, like Euro-Dollar [1]. The biggest, the most efficient and dynamic financial market in the world with turnover of more than 2.5 trillion dollars is called exchange market or so called Forex. Forex is an international market, its main elements are banks but some opportunities are provided for entering investors, minor customers and ext with the help of brokers [2]. Shuo Yao, Michael Pasquier and Chai Queck managed portfolios of various exchanges using fuzzy neural networks, and made the fund return to $47.39 \%$ which than previous effort had increase to 1.4\%. Buy and sell signals were published separately for each share and by combining with moving average indicator, finally buy and sell order published [3]. Using a genetic algorithm, Adam Bicz created a profitable strategy in Forex market in which two trees of decision making are used for opening short-term and long-term trading which are used in euro-dollar symbol which each of them determine border level of technical indicator by a logical operator [4].

The model suggested is one of the latest trading systems based on fuzzy which so far has not had similar model and that most of trading systems work according to individual experiments and manually. So by providing the system for the first time can be provided the groundwork for continuous improvement of fuzzy systems for commerce.

\section{Forex}

Forex is in fact buying exchange and selling another exchange in a virtual integrated market in which currencies are traded in pair. Since all the nations are going toward economic globalization, the activity of the market will be continued and never stops.

\section{Trading strategy}

As a trader, we need to have a technique for buy and sell. Based on definitions present, in capital market there are two types of general strategy: technical analysis and fundamental analysis.

Technical analysis is investigating price movement. In this analysis, future price can be predicted using chart behavior. By analyzing charts, traders can recognize the patterns and trends and they help traders to recognize good trading opportunities. Using indicators in this type of analyzing is so common $[5,6]$.

Fundamental analysis is a method for observing market from aspects of economic, social and political forces which influence on supply and demand.

Using technical analysis, in this article we tried to predict behavior of euro-dollar. One of efficient meaning in this field is fuzzy logic. By using this logic, chart patterns and trends can be modeled and they are extracted based on fuzzy rules which are buying and selling signals and according to them trading can be done.

\section{Types of price charts}

The most important charts in technical analyzing are bar, linear and candle. In this article the latter is used. Candle charts show bar data in a nicer graphic. According to figure 1, candle charts are formed of two parts of body and shadow. Body is length of opening rate and its closing and shadow indicates distance of the highest price to rate of closing and the least price to rate of opening.

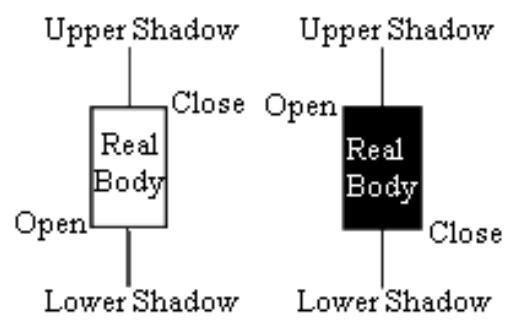

Figure 1: candle chart in exchange market

There is not a significant difference between bar chart and candle one. The only difference is their body which is shown in candle chart as a rectangle [7]. 


\section{Modeling Candle lines}

In a candle chart, length of shadows and body have important role in recognizing candlestick pattern and they determine their efficiency. Describing a candlestick line is not clear like, high, short or average. No certain border is for defining length of body or shadow. In this method four linguistic variables are used (neutral, short, average and long) for differentiating fuzzy sets of shadows and length of body. Figure 2 shows membership function of the variables. Length of body and shadows are determined in range $(0 \%$ and $4 \%)$ which indicate percent of daily volatilities and body length and shadows vary in different market conditions. They can be modeled with three yardstick of long, short and average. Membership function of them is shown in the figure 2.

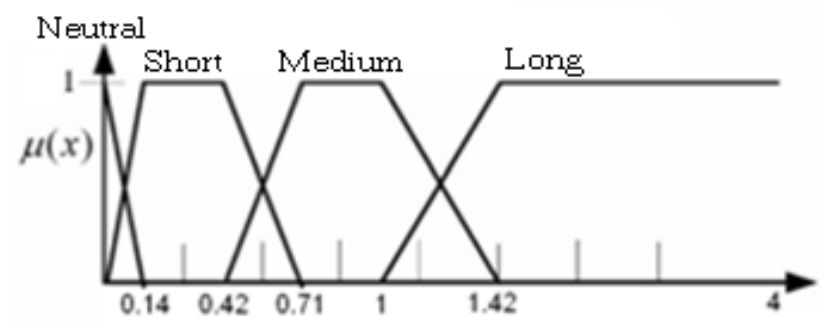

Figure 2: Membership function of body length and shadows

Values of daily volatilities can be calculated based on relationship 1:

$\mathrm{L}_{\text {upper shadow }}=\left(\frac{\left[\text { high }-\max \left(\text { open }_{y} \text { close }\right)\right]}{\text { open }}\right)$

$\mathrm{L}_{\text {lowershadow }}=\left(\frac{\left[\min \left(\text { open }_{y} \text { close }\right)-\text { low }\right]}{\text { open }}\right)$

$\mathrm{L}_{\text {body }}=\left(\frac{\left[\max \left(\text { open, }_{,} \text {close }\right)-\min \left(\text { open, }_{\text {close }}\right)\right]}{\text { open }}\right)$

$\mu_{\text {Long }}=\left\{\begin{array}{cr}0 & x<a \\ \frac{x-a}{b-a} & a \leq x \leq b \\ 1 & x>b\end{array}\right\}$

Parameters of the function are defined as follows:

Function of short membership $=(0,0.14,0.42,0.71)$

Function of average membership $=(0.42,0.71,1,1.42)$

Function of long membership $=(1,1.42)$ 
Color of body is also a key characteristic of a candle and it is defined with three words of black, white and neutral. If price of opening be more than price of closing, white definition is used. If rate of opening be less than rate of closing, black definition is used and finally if price of opening be equal to rate of closing, neutral definition is used.

Definition of body color is determined in form of fuzzy rules according to relation 3 :

if close > open, then the body colour is BLACK

if close < open, then the body colour is WHITE

if close $=$ open, then the body colour is CROSS

\section{Fuzzification}

A fuzzy inference system is formed of three general steps:

1. Fuzzification

2. Using fuzzy rules and relations

3. Defuzzification

In the first step of the system we need to fuzzificate data and rules .In this article triangular and trapezoidal functions which used most common, applied that have acceptable effects on system accuracy.

Only by defining length of body and shadows of a candle, it cannot be modeled. Model of body and shadows length shows only appearance of a candlestick line. For inserting a candle in self place, the relationship with previous candle should be specified. Two features are needed for modeling relations between candles: condition of opening and closing. Relation of price of opening and closing to previous candle is used for modeling condition of opening and closing. In figure 3 , following candle shows chart of trading price of the previous day. Unit of axis X is trading prices of previous day and unit of axis y is value of membership function.

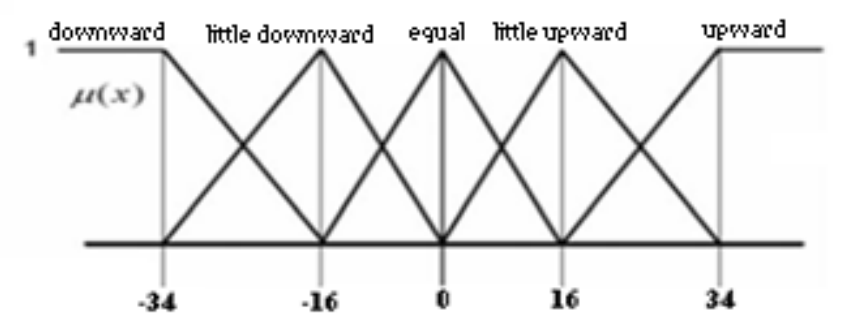

Figure (3): membership function of opening and closing condition

Five linguistic variables are defined for relation of opening condition:

Downward closing, little downward closing, equal opening, little upward opening, upward opening. Five linguistic variables are defined for relation of closing condition:

Downward closing, little downward closing, equal closing, little upward closing, upward closing. The function used for condition (downward opening and downward closing) is in form of relation 4:

$\mu_{\text {downward opening and closing }}=\left\{\begin{array}{cr}0 & x<a \\ \frac{x-a}{b-a} & a \leq x \leq b \\ 1 & x>b\end{array}\right\}$

The function used for condition ((upward opening and upward closing)) is in form of relation 5: 


$$
\mu_{\text {upward opening and closing }}=\left\{\begin{array}{cc}
1 & x<a \\
\frac{b-x}{b-a} & a \leq x \leq b \\
0 & x>b
\end{array}\right\}
$$

Membership function for other conditions (little downward opening and little downward closing, equal opening or equal closing, little upward opening or little upward closing, upward opening or upward closing)) is defined in triangle form in relation 6.

$\mu_{\text {other conditions }}=\left\{\begin{array}{cr}0 & x<a \\ \frac{x-a}{b-a} & a \leq x \leq b \\ \frac{c-x}{c-b} & b \leq x<c \\ 0 & x \geq c\end{array}\right\}$

Linguistic variable parameters of conditions of opening and closing are determined by price of previous candle. For example, if opening price in a desired time period be equal with the least opening or closing price of the previous candle, then a little downward opening condition occurs and if closing price be equal to highest Price or closing price of previous candle, then a little upward closing condition occurred. By combining descriptions of candles and the relations between them, a candle is defined fully. Length of body and shadows model appearance and opening or closing conditions model casual relations and candlestick are main part of price patterns.

\section{Modeling of bulling or bearish trend}

We used one linguistic variable for modeling of medium-term trend. 4hour timeframe used for showing trend in price patterns. Five linguistic variables are used for defining trend: neutral, weak, medium, strong and very strong. Membership functions of neural, weak, medium and strong trend are Gaussian's type. Also membership function of very strong trend is Sigmoid's type which is shown in figure 4 . Relation 7 illustrates computation of trend's type.

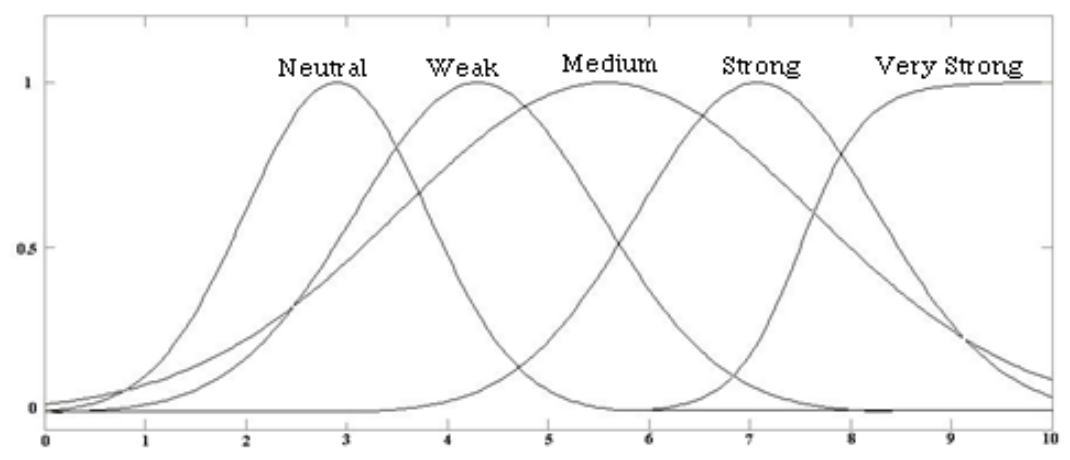

Figure 4: membership function of trend

$\mathrm{TS}=\sqrt[n]{100-\frac{100}{1+10^{4} \sum_{i=1}^{\mathrm{m}}|\operatorname{close}(\mathrm{i})-\operatorname{open}(\mathrm{i})|}}$

Where ${ }^{m}$ and $n$ are respectively period of indicator's computation and computation cycle of market's type [8].

\section{Programming languages}


According to rules mentioned for defining fuzzy variables, we can make rules mentioned in form of a program. Nowadays three are different programming languages some of which are given here:

PASCAL, BASIC, FORTRAN, GASP, GPSS, SLAM, MATLAB, MQL

MQL programming language is like C program in which goals like preparing expert, indicator or script is followed. The language cannot be performed in windows operation system like other programming languages and it can be used only in Metatrader. In this research above language was used for preparing indicator. According to fuzzy rules mentioned in previous sections, they can be written in MQL languages and it can be read again in Metatrader environment on the chart after compiling.

\section{Fuzzy indicator}

After completing programming level successfully and trading strategy`s being an indicator it is enough to use it by placing it in special places of Metatrader software or any type of other trading platform. In this article trading strategy is in form of an indicator and we bring it into Metatrader software. By performing indicator on euro-dollar chart in 4-hour time frame, buy and sell signals can be seen. Red color means selling and blue color is buying. So changing indicator color will be sign of trend changing. In figure 5 can be seen buy and sell point by means of desired indicator.

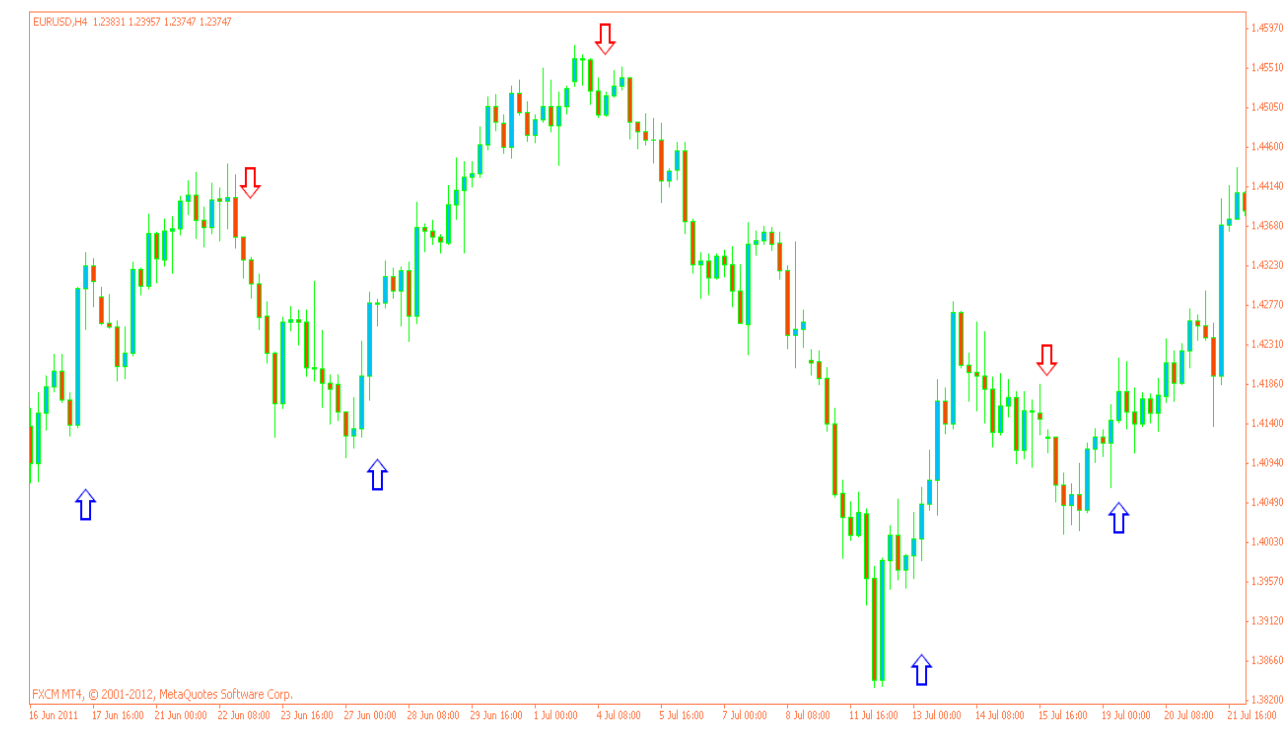

Figure (5): performing indicator on Euro-dollar chart

\section{Indicator test}

Since indicator was designed for Euro-dollar charts, the same part of exchange should be used for testing. Framework time is 4 hours. Trading was done on a $10000 \$$ account. Results from 20.1.2011 to 22.12.2011 are shown in figure 6 which indicate of earned profit.

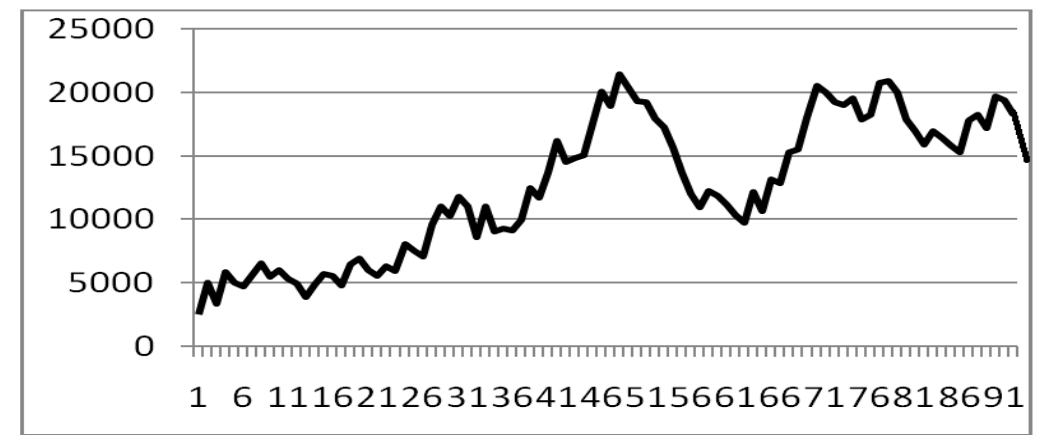

Figure (6): chart of earned profit due to effect of predicting indicator 


\section{Conclusion}

In this article an auto trading system was invented based on fuzzy logic and technical analysis which according to it signals of buy and sell can be created and then trading can be started. It is worth mentioning that financial markets have the most investment risk and definitely the profit gained should be compared with other fields of investment and then be invested by a suitable and logic analysis. Certainly the system can be used for an unskilled person who does not have enough skill in financial markets but for more success in this field, having enough science and experience is recommended. The invented system should be used as a consulter in this field. Due to predictions of the system, profit of \%143.8 was gained for 2011price data of euro- dollar and according to level of risk it seems logical. Du to earned yield of desired model, it can be used for credit and financial institutes, banks or investment companies that it improved trading system over the world.

\section{References}

[1] A. Mohammadi, Meeting international markets of exchange, Azadi Publication, (2010).

[2] M. Kia, Fuzzy logic in Matlab, Tehran, Kianrayane sabz Publication, 2010.

[3] Y. Shuo and M. Pasquier and C. Quek, A Foreign Exchange Portfolio Management Mechanism Based On Fuzzy Neural Networks, Singapore, IEEE Congress on Evolutionary Computation,(2007).

[4] A. Bicz Evolutionary Algorithm in Forex Trade Strategy Generation, International Multi conference On Computer Science and Information Technology, (2008).

[5] R. Colby, The Technical Market Indicator, Wall Street courier, (2005).

[6] R. Edwards, Currency trading for a Living in forex's Market, Trading Intl, (2004).

[7] D. Richard, Technical analyses Application in the Global Currency market, New York Institute of Finance-New York, (2002).

[8] M. Owski, Attacking Currency Trends: How to Anticipate and Trade Big Moves in the currency market, Wiley Trading, (2011). 